\title{
Muscle Kinetics and Exercise: Any Advantages of Dietary Proteins?
}

\section{El Khoury Dalia*}

Department of Nutritional Sciences, Faculty of Medicine, University of Toronto, Toronto, ON, M5S 3E2, Canada

It is well established that macronutrients and micronutrients modulate responses of muscle kinetics, fatigue and immunity to exercise, although nutritional information have been extremely variable in the sports field. Dietary protein has gained a particular interest in the past few decades with respect to its role in exercise performance and recovery. Amongst the distinct functions of proteins and amino acids in exercise, a superior role in promoting a positive muscle protein balance, characterized with increased muscle protein synthesis and attenuated muscle protein breakdown, was highlighted by numerous studies [1]. A special emphasis on resistance exercise was noted in the majority of these studies, because muscular anabolic responses and muscle damage have been described following high intensity resistance exercise to a greater extent than moderate intensity endurance training.

As a means to maximize the anabolic effects of resistance training on skeletal muscles, muscular pool and circulating levels of amino acids should be always maintained. The ingestion of dietary protein and/or amino acids at adequate levels alongside resistance training has been proven to be an effective way to increase protein synthesis rates and consequently skeletal muscle hypertrophy over time. Although ingestion of essential and non-essential amino acids increases plasma amino acid concentrations up to 3 hours, the availability of essential amino acids is the primary promoter of muscle protein synthesis [2]. Studies have demonstrated greater increases in muscle protein synthesis rates when essential amino acids were ingested after resistance exercise rather than when essential amino acids ingested at rest or when resistance exercise performed in the fasting state [3]. However, it seems that a threshold exists above which essential amino acids do not induce further increments in protein synthesis. In the study of Tipton et al. [4], ingestion of either $40 \mathrm{~g}$ of essential amino acids or $40 \mathrm{~g}$ of a mixed amino acid mixture composed of $21.4 \mathrm{~g}$ of essential amino acids an hour after a resistance exercise bout similarly increased amino acid concentrations by 2.5 -fold and protein synthesis by $70 \%$.

Skeletal muscle kinetic responses were also found to depend on the quality of ingested dietary proteins. Consumption of various types of proteins was found to affect differently the amplitude and possibly the duration of muscle protein synthesis after resistance exercise [5]. Dairy proteins appeared to be more beneficial during high intensity resistance training when compared to other protein types. Milk proteins promoted greater protein accretion in comparison to an isonitrogenous and isoenergetic soy protein when ingested after resistance training in healthy young men [6]. With the immediate provision of $25 \mathrm{~g}$ of whey protein, a major protein constituent of milk, following resistance exercise, a sustained enhancement of myofibrillar protein synthesis and anabolic intramuscular signaling responses was reported up to 3-5 hours in recreationally active men [7]. Even as part of a whole-food dairy product, the benefits of whey proteins in enhancing resistance exercise-induced muscle anabolism have been shown. After 45-min of a bout of endurance exercise, the consumption of fat-free chocolate milk revealed unique benefits including enhanced muscle protein fractional synthetic rate, reduced whole body proteolysis, improved skeletal muscle protein turnover and increased time to exhaustion compared to a non-nitrogenous isocaloric carbohydrate control beverage in male runners [8]. Dairy proteins are rich sources of branched-chain amino acids including leucine, isoleucine and valine, which are among the few amino acids that can be actively metabolized in the skeletal muscle during exercise [9]. The anabolic stimulus of branched-chain amino acids on muscle protein kinetics has been mainly attributed to leucine [10], although the importance of leucine relative to the other essential amino acids in regulating muscle kinetics is still not clear. The benefit of leucine in stimulating muscle protein synthesis was consistently established in well-controlled cell culture and animal studies [11]. Animal studies have shown that leucine can independently stimulate muscle protein synthesis by phosphorylating proteins involved in mRNA translation initiation such as through the mammalian target of rapamycin (mTOR) signaling cascade [12], a critical mediator of both contraction- and essential amino acidsmediated increase in muscle protein synthesis [13-14]. However, inconsistencies in findings were reported in humans. In the study of Glynn et al. [15] on young men and women, the consumption of an essential amino acid mixture $(10 \mathrm{~g})$ containing a higher concentration of leucine $(3.5 \mathrm{~g})$ did not improve net protein anabolism beyond that of the mixture containing the lower amount of leucine $(1.85 \mathrm{~g})$ under resting conditions. Furthermore, compared to an isonitrogenous whey protein drink providing an adequate mixture of essential amino acids ( $69 \mathrm{~g}$ total protein containing $4.7 \mathrm{~g}$ leucine), the addition of leucine to the whey protein drink ( $69 \mathrm{~g}$ total protein containing $17.6 \mathrm{~g}$ leucine) failed to further enhance muscle protein synthesis when consumed over a 6-hour period after performing a 30-minute bout of moderate exercise in older adults [16]. In the study of Churchward-Venne et al. [17], a relatively small amount of leucine $(0.75 \mathrm{~g})$ was found to be sufficient to achieve maximal stimulation of muscle protein synthesis when other essential amino acids were provided in larger quantities $(8.5 \mathrm{~g})$ following a bout of resistance exercise. Thus, at rest and during recovery from resistance exercise, an essential amino acid profile at adequate doses and of high quality can maximally stimulate muscle protein synthesis with any added leucine providing no further benefits. Caution must be taken since leucine was always supplemented as part of an amino acid mixture. It should be thus clarified whether leucine could be beneficial if used alone or whether its effects on protein balance regulation are linked to the presence of other essential amino acids in the same amino acid mixture. In addition, it is still unclear whether the intracellular or the extracellular availability of leucine and/ or essential amino acids is the major contributor to muscle protein synthesis regulation. Although some studies showed that postprandial stimulation of muscle protein synthesis is directly proportional only to the rise in blood leucine [18], others found that peak activation

*Corresponding author: El Khoury Dalia, Department of Nutritional Sciences, Faculty of Medicine, University of Toronto, Toronto, ON, M5S 3E2, Canada; Tel. 1-416-978-0799; Fax: 1-416-978-5882; E-mail: dalia.elkhoury@utoronto.ca

Received December 17, 2012; Accepted December 19, 2012; Published December 21, 2012

Citation: Dalia EK (2013) Muscle Kinetics and Exercise: Any Advantages of Dietary Proteins? Single Cell Biol 2: e117. doi:10.4172/2168-9431.1000e117

Copyright: (c) 2013 Dalia EK. This is an open-access article distributed under the terms of the Creative Commons Attribution License, which permits unrestricted use, distribution, and reproduction in any medium, provided the original author and source are credited. 
of muscle protein synthesis is not necessarily driven by leucinaemia [17]. Furthermore, amino acid transport across the sarcolemma [19] and intracellular availability of amino acids [20] were also reported to control muscle protein synthesis.

Exercise-induced skeletal muscle injury manifests in the minutes to days following an acute exercise bout and is accompanied by several signs including muscle pain, delayed-onset muscle soreness and impaired physical performance. Although aerobic exercise can cause muscle damage, the extent of damage is often less with lower intensity and longer duration aerobic training sessions in comparison to higher intensity shorter duration resistance exercise bouts [21]. An adequate muscle recovery was found to minimize symptoms of exerciseinduced muscle damage. Anti-catabolic properties of branched-chain amino acids have been revealed in various studies, when consumed immediately before, during and/or after exercise sessions. When 100 $\mathrm{mg} / \mathrm{kg}$ body weight of branched-chain amino acids were ingested 15 min before exercise, immediately before exercise, at 15, 30, 45 and 60 min during exercise and at 15, 30, 60 and 90 min of recovery, a reduced release of $32 \%$ of aromatic amino acids, tyrosine and phenylalanine, from the legs was described during the 2-hour recovery period [22]. Furthermore, when the recommended intake of branched-chain amino acids ( $12 \mathrm{~g} /$ day) was consumed for 14 days, decrease in serum concentrations of indicators of muscle damage, creatine kinase and lactate dehydrogenase, were observed following a cycling session of 120 minutes [23]. On the other hand, the role of other essential amino acids in modulating muscle damage in response to exercise has been rarely investigated and needs to be defined.

There is still no agreement regarding the exact protein recommendations for exercising individuals and athletes for the generation of optimal muscle kinetic responses to exercise. While some studies consider protein intakes above the recommendations for the general population $0.8 \mathrm{~g} / \mathrm{kg} /$ day crucial for individuals engaged in physical activity and exercise [24-26], others are convinced that those individuals are just required to adopt a well-balanced diet adequate to meet their protein needs that match those of the general population [27]. In vulnerable groups including low energy consumers such as gymnasts and those restricting energy intake to adjust the weight for competition, vegetarian athletes with restricted choice, young performers who rely on nutritionally-poor school meals as the main meal of the day and student athletes who are living on limited budget, increased protein intakes through a combination of foods and dietary supplements may be though needed.

Although the exact protein needs of exercising individuals and athletes are not yet clearly defined, existing literature supports an essential role of proteins and amino acids in promoting muscle hypertrophy and muscle recovery if consumed at least at the recommended amounts and as part of a healthy balanced diet with an adequate profile of essential amino acids consistent with high quality proteins. Knowing that the signaling cascades for muscle kinetics are complex, an understanding of the exact mechanisms through which these pathways respond to exercise and to dietary protein interventions is essential and should be further explored. These findings would provide sports scientists and coaches with evidence-based information to maximize training adaptations.

\section{References}

1. Poole. C, Wilborn. C, Taylor. L, Kerksick. C (2010) The role of post-exercise nutrient administration on muscle protein synthesis and glycogen synthesis. $J$ Sports Sci Med 9: 354-363.

2. Volpi E, Kobayashi H, Sheffield-Moore M, Mittendorfer B, Wolfe RR (2003)
Essential amino acids are primarily responsible for the amino acid stimulation of muscle protein anabolism in healthy elderly adults. Am J Clin Nutr 78: 250258.

3. Drummond MJ, Dreyer HC, Fry CS, Glynn EL, Rasmussen BB (2009) Nutritional and contractile regulation of human skeletal muscle protein synthesis and mTORC1 signaling. J Appl Physiol 106: 1374-1384.

4. Tipton KD, Ferrando AA, Phillips SM, Doyle D Jr, Wolfe RR (1999) Postexercise net protein synthesis in human muscle from orally administered amino acids. Am J Physiol 276: E628-634.

5. Tang, J.E., S.M. Phillips (2009) Maximizing muscle protein anabolism: the role of protein quality. Curr Opin Clin Nutr Metab Care 12: 66-71.

6. Wilkinson SB, Tarnopolsky MA, Macdonald MJ, Macdonald JR, Armstrong D, et al. (2007) Consumption of fluid skim milk promotes greater muscle protein accretion after resistance exercise than does consumption of an isonitrogenous and isoenergetic soy-protein beverage. Am J Clin Nutr 85: 1031-1040.

7. West DW, Burd NA, Coffey VG, Baker SK, Burke LM, et al. (2011) Rapid aminoacidemia enhances myofibrillar protein synthesis and anabolic intramuscular signaling responses after resistance exercise. Am J Clin Nutr 94: 795-803

8. Lunn WR, Pasiakos SM, Colletto MR, Karfonta KE, Carbone JW, et al. (2012) Chocolate milk and endurance exercise recovery: protein balance, glycogen, and performance. Med Sci Sports Exerc 44: 682-691.

9. Rennie MJ, Bohé J, Smith K, Wackerhage H, Greenhaff P (2006) Branchedchain amino acids as fuels and anabolic signals in human muscle. J Nutr 136: 264S-268S.

10. Drummond MJ, Rasmussen BB (2008) Leucine-enriched nutrients and the regulation of mammalian target of rapamycin signalling and human skeletal muscle protein synthesis. Curr Opin Clin Nutr Metab Care 11: 222-226.

11. Pasiakos SM, McClung JP (2011) Supplemental dietary leucine and the skeletal muscle anabolic response to essential amino acids. Nutr Rev 69: 550-557.

12. Crozier SJ, Kimball SR, Emmert SW, Anthony JC, Jefferson LS (2005) Oral leucine administration stimulates protein synthesis in rat skeletal muscle. J Nutr 135: $376-382$

13. Dickinson, J.M, Fry C.S, Drummond M.J, Gundermann D.M, Walker D.K, et al. (2011) Mammalian target of rapamycin complex 1 activation is required for the stimulation of human skeletal muscle protein synthesis by essential amino acids. J Nutr 141: 856-862.

14. Drummond, M.J, Fry C.S, Glynn E.L, Dreyer H.C, Dhanani S, et al. (2009) Rapamycin administration in humans blocks the contraction-induced increase in skeletal muscle protein synthesis. J Physiol 587: 1535-1546.

15. Glynn, E.L, Fry C.S, Drummond M.J, Timmerman K.L, Dhanani. S, et al. (2010) Excess leucine intake enhances muscle anabolic signaling but not net protein anabolism in young men and women. J Nutr 140: 1970-1976.

16. Koopman,R, Verdijk.L.B, Beelen.M, Gorselink.M, Kruseman.A.N, et al. (2008) Co-ingestion of leucine with protein does not further augment post-exercise muscle protein synthesis rates in elderly men. Br J Nutr 99: 571-580.

17. Churchward-Venne.T.A., Burd.N.A, Mitchell.C.J, West.D.W, Philp.A, et al. (2012) Supplementation of a suboptimal protein dose with leucine or essential amino acids: effects on myofibrillar protein synthesis at rest and following resistance exercise in men. J Physiol 590: 2751-2765.

18. Norton, L.E, Layman D.K, Bunpo.P, Anthony A. Brana G.D.V, et al. (2009) The leucine content of a complete meal directs peak activation but not duration of skeletal muscle protein synthesis and mammalian target of rapamycin signaling in rats. J Nutr 139: 1103-1109.

19. Hundal HS, Taylor PM (2009) Amino acid transceptors: gate keepers of nutrient exchange and regulators of nutrient signaling. Am J Physiol Endocrinol Metab 296: E603-613.

20. Biolo G, Maggi SP, Williams BD, Tipton KD, Wolfe RR (1995) Increased rates of muscle protein turnover and amino acid transport after resistance exercise in humans. Am J Physiol 268: E514-520.

21. Bloomer, R.J. (2007) The role of nutritional supplements in the prevention and treatment of resistance exercise-induced skeletal muscle injury. Sports Med 37: $519-532$

22. Blomstrand E, Saltin B (2001) BCAA intake affects protein metabolism in 
muscle after but not during exercise in humans. Am J Physiol Endocrinol Metab 281: E365-E374

23. Coombes JS, McNaughton LR. (2000) Effects of branched-chain amino acid supplementation on serum creatine kinase and lactate dehydrogenase after prolonged exercise. J Sports Med Phys Fitness 40: 240-246.

24. Tarnopolsky M (2004) Protein requirements for endurance athletes. Nutrition 20: $662-668$.
25. Tarnopolsky MA, Atkinson SA, MacDougall JD, Chesley A, Phillips S, et al. (1992) Evaluation of protein requirements for trained strength athletes. J App Physiol 73: 1986-1995.

26. Lemon, PW. (1994) Protein requirements of soccer. J Sports Sci 12: S17-S22.

27. Tipton KD, Wolfe RR (2004) Protein and amino acids for athletes. J Sports Sci; 22: 65-79. 